\title{
Nutrientes y renovación del agua en aiguamolls de l'Empordà. (NE de la península lbérica). Uso potencial de agua residual para la inundación de zonas húmedas
}

\author{
Xavier Quintana, Lluís Suñer, Rosa Trobajo, Stephanie Gascón y Ramón Moreno-Amich. \\ Instituto de Ecología Acuática y Depto. de Ciencias Ambientales. Universidad de Girona. Campus de Montilivi. \\ 17071 Girona, Catalunya, España.
}

\section{RESUMEN}

De septiembre de 1996 a agosto de 1997 se llevó a cabo el seguimiento de la calidad ecológica del agua del Parque Natural de "Aiguamolls de l'Empordà". El objetivo de este estudio es la evaluación del impacto del efluente procedente de una planta de tratamiento de aguas residuales próxima, en la zona alargada de la marisma del Parque Natural. En este artículo presentamos las características físicas y químicas y la variación temporal de la concentración de nutrientes en el sistema lenítico de "Aiguamolls de l'Empordà", como un paso previo en la evaluación del efecto de la agua residual depurada en el sistema de la marisma salada. Se analizó la variación zonal de la concentración de nutrientes y su relación con la tasa de renovación y el origen del agua. Los resultados muestran variaciones significativas de la relación N/P que está relacionada con la tasa de renovación del agua. Sistemas confinados presentan valores bajos para esta relación. Teniendo en cuenta la concentración de nutrientes del agua depurada (rica en nitrógeno), se recomiendan zonas inundadas con elevadas tasas de renovación del agua y un elevado valor de la relación N/P.

Palabras clave: humedales mediterráneos, seguimiento, relación nitrógeno/fósforo, tasa de renovación, regulación de flujos.

\begin{abstract}
The monitoring of the ecological water quality of "Aiguamolls de l'Empordà" Natural Park was carried out between September 1996 to August 1997. The aim was to evaluate the impact of effluent from a nearby wastewater treatment plant, on the flooding of marshes in the Natural Park. In this paper we present physical and chemical data together nutrient concentrations and its changes in the lentic system of the "Aiguamolls de l'Empordà". The ultimate aim was to evaluate the effect of reclaimed wastewater on the salt marsh system. Seasonal variation in nutrients concentrations and their relationship to turnover and source of water were analysed. Significant variation in the N/P ratio was related to water turnover. Confined systems presented lower values for this ratio. Taking into account the nutrient concentrations of the reclaimed water, which is rich in nitrogen, it is recommended flooding applied in zones with high water turnover and higher N/P ratios.
\end{abstract}

Keywords: Mediterranean wetlands, monitoring, nitrogen/phosphorus ratio, turnover rate, flux regulation.

\section{INTRODUCCIÓN}

La zona conocida como Aiguamolls de l'Empordà ocupa el litoral de la Bahía de Roses, en el límite norte de la costa mediterránea ibérica. Se trata del frente de una llanura deltaica, originada por los aportes de los ríos Muga y Fluvià y está formada por un conjunto de lagunas costeras, marismas mediterráneas (no influidas por la marea) y sus zonas inundables (ver Fig. 1 de Moreno-Amich et al., pag. 18).

En 1984 se creó el Parque Natural de Aiguamolls de l'Empordà (en adelante, PNAE). Desde su creación la gestión del PNAE ha ido encami-

Limnetica 16: 49-59 (1999)

(C) Asociación Española de Limnología, Madrid. Spain. ISSN: 0213-8409. 
nada a "devolver a estas zonas húmedas su antigua extensión y productividad" (Sargatal, 1989), compensando las considerables pérdidas por desecación que ha sufrido la zona durante las últimas décadas. El objetivo principal hasta la actualidad ha sido incrementar las poblaciones de aves tanto sedentarias como visitantes, aumentando la disponibilidad de hábitats acuáticos para las aves mediante el incremento de la superficie inundada. En este sentido se construyó una laguna artificial ("Estany del Cortalet") alimentada con agua procedente del "Rec Corredor".

De todas las actuaciones quizás la más remarcable por sus consecuencias ha sido la instalación de una compuerta en el canal de desagüe MassonaRec Sirvent, que entró en funcionamiento en 1990. Con la compuerta se puede ahora retener dentro de la reserva el agua dulce superficial que circula por el "Rec Corredor", hasta alcanzar un nivel medio de agua de $0.8 \mathrm{~m}$ sobre el nivel del mar durante los períodos de inundación, y ha permitido aumentar considerablemente la superficie inundada (antes, en inviernos normales, era de 37 ha y ahora supera las 200 ha). Disponemos de estudios realizados en el momento de la instalación de la compuerta, que demuestran los cambios en la calidad del agua y en la composición de las comunidades acuáticas planctónicas causadas por la puesta en marcha de dicha compuerta (Quintana, 1995; Quintana et al., 1998a; Quintana et al., 1998b). Además, desde 1996 venimos realizando una monitorización de la calidad ecológica de las aguas del PNAE, a partir de la cual se ha observado un aumento del tiempo de permanencia del agua dulce y de la superficie afectada por la inundación. También la regulación de flujos ha causado un aumento paulatino del grado de eutrofia (Moreno-Amich et al., 1998), debido a la mayor aportación de nutrientes.

Por otro lado, en el territorio del PNAE se ha construido una depuradora de aguas residuales (EDAR) de tipo mixto, con un tratamiento de fangos activados con aireación prolongada sin decantación primaria, seguido por un sistema de lagunaje con una primera laguna de sedimentación, donde los fangos se mineralizan, y una segunda laguna de afinamiento, donde se produce la última fase de depuración y desinfección de forma natural. Posteriormente se le ha añadido un sistema de tratamiento adicional del agua depurada mediante un sistema de humedales construidos (SHC), con el objetivo de reducir los niveles de nutrientes del efluente final de la EDAR. Se espera que el efluente de la EDAR sea apto para la creación y restauración de zonas húmedas en el PNAE sin que su utilización suponga un incremento en la eutrofización de la marisma. En relación a esta actuación se ha iniciado un programa de vigilancia ambiental para constatar que el diseño y funcionamiento del SHC son correctos y no se produce eutrofización.

Los datos presentados muestran el efecto a nivel espacio-temporal de la regulación de flujos sobre la composición de nutrientes, así como la conveniencia de la utilización del efluente de la EDAR en el PNAE. El objetivo de este trabajo es evaluar la viabilidad de la utilización del agua residual depurada para la inundación de las zonas húmedas naturales de Aiguamolls de l'Empordà, estableciendo los lugares y períodos en los que la inundación es aceptable y evitar los vertidos de agua depurada en aquellas zonas donde pueda causar efectos no deseables. Para ello préviamente debe establecerse la dinámica temporal de la composición de nutrientes de la marisma y analizar, desde el punto de vista de los nutrientes, los efectos producidos por la regulación de flujos.

\section{MATERIAL Y MÉTODOS}

El estudio se ha realizado en la marisma del parque natural Aiguamolls de l'Empordà durante el período comprendido entre el mes de septiembre de 1996 y agosto de 1997. Se ha muestreado con frecuencia semanal seis depresiones inundables de la marisma y el canal que alimenta con agua dulce la zona ("Rec Corredor"). Las depresiones fueron seleccionadas en base a la distancia de estas al "Rec Corredor", quedando agrupados en dos sectores: En el sector sur, muy próximo al punto de entrada de agua dulce, se encuentran la Rogera y la Riereta, dos lagunas costeras conectadas entre si, de inundación permanente (o semipermanente en el caso de la Riereta). En el sector 
norte, más alejado de la fuente de agua dulce, se encuentran una serie de pequeñas depresiones que sufren desecación estival (en adelante llamadas Tamariu, Conectada y Litoral), y una laguna permanente o semipermanente de mayores dimensiones llamada Estany d'en Túries.

Se han medido en el campo la temperatura, la conductividad $\left(\mathrm{EC}_{25}\right)$ el $\mathrm{pH}$, y el de oxigeno disuelto y se han recogido aguas filtradas in situ (Whatman GF/C) para el análisis de amonio, nitrito, nitrato y fósforo reactivo soluble (SRP). El filtro se recogió para el análisis de clorofila.

Los análisis de $\mathrm{NO}_{2}^{-}, \mathrm{NO}_{3}^{-}$y SRP se realizaron mediante FIA (flow injection analysis) según los métodos descritos en Ruzicka \& Hansen (1981) y Cerdá et al. (1996). El $\mathrm{NH}_{4}{ }^{+}$se analizó siguiendo a Solórzano (1969). El caudal del "Rec Corredor" se midió mediante correntímetro. La extracción de clorofila se realizó utilizando metanol $90 \%$ como disolvente. El contenido de clorofila a se midió después de 20-24 horas siguiendo las expresiones de Talling \& Driver (1963).

\section{RESULTADOS}

La hidrología de la marisma de "Aiguamolls del Empordà", queda reflejada en la evolución temporal de los valores de la conductividad. El aumento de esta el 10 de diciembre en todos los puntos (Fig.

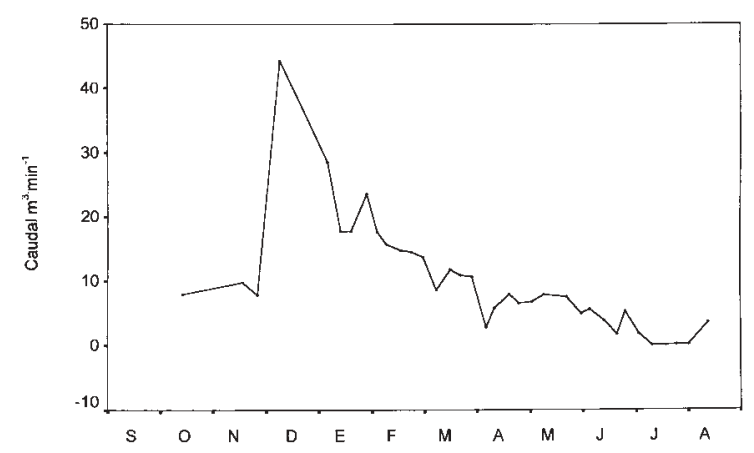

Figura 1. Variación temporal del caudal $\left(\mathrm{m}^{3} \mathrm{~min}^{-1}\right)$ del "Rec Corredor" en 1996/1997. Temporal variation in flow $\left(\mathrm{m}^{3} \cdot \mathrm{min}^{-1}\right)$ of the "Rec Corredor" in 1996/1997.
2) se debe a la entrada masiva de agua durante el temporal de mar. Posteriormente, la conductividad disminuye paulatinamente debido a la entrada de agua dulce procedente del "Rec Corredor" y desviada por efecto de la compuerta. A partir de este momento se observan dos comportamientos distintos en la evolución de la conductividad. Los puntos del sector sur, más cercanos al punto de entrada de agua dulce, mantienen unas conductividades que oscilan entre 7 y $13 \mu \mathrm{S} \cdot \mathrm{cm}^{-1}$, mientras que en los puntos más alejados (sector norte) las conductividades aumentan progresivamente hasta superar los $40 \mathrm{mS} \mathrm{cm}^{-1}$ cuando la entrada de agua dulce es poco importante, coincidiendo con valores bajos del caudal del "Rec Corredor") (Fig. 1). En el sector sur, la dulcificación del sistema es más patente y prolongada en el tiempo, debido a su proximidad a la fuente de agua dulce.

Las aguas del "Rec Corredor", tienen una concentración media de nitrógeno inorganico disuelto superior a $8000 \mu \mathrm{gL}^{-1}$ (Tabla 2), principalmente en forma de nitrato. Los valores de nitrato fluctuan poco durante todo el año (Fig. 3) a excepción de la época estival en la que se producen disminuciones de su concentración, probablemente debidas a la desnitrificiación, coincidiendo con los mínimos valores de caudal (Fig. 1). La relación DIN/SRP de este punto es siempre muy elevada, a pesar de contener unas concentraciones de SRP relativamente elevadas.

Las concentraciones máximas de nitrógeno inorgánico disuelto observadas en los puntos de la marisma, coinciden con los momentos de entrada de agua al sistema. Estas aportaciones de nitrógeno, pueden ser puntuales, coincidiendo con los temporales de mar (10 de diciembre) (Figs. 3 y 4), o con lluvias aisladas como las de principios de verano. También pueden ser aportaciones continuas como la asociada a la entrada de agua dulce desviada a la marisma, durante los meses de invierno, debido al funcionamiento de la compuerta, coincidiendo con valores altos de caudal del "Rec Corredor" (Fig. 1). Esta entrada explicaría el excedente de nitrato observado en todos los puntos durante dicho período (Figs. 3 y 4). El excedente de nitrato es más aparente y prolongado en el tiempo en los puntos del sector sur, 
Table 1. Descriptores estadísticos de los nutrientes inorgánicos disueltos en los puntos muestreados. Descriptors of dissolved inorganic nutrients in sampling sites.

\begin{tabular}{|c|c|c|c|c|c|c|c|c|}
\hline & \multicolumn{4}{|c|}{$\mathbf{N H}_{4}{ }^{+}\left(\boldsymbol{\mu g} \mathbf{N} \cdot \mathbf{L}^{-1}\right)$} & \multicolumn{4}{|c|}{$\mathrm{NO}_{2}^{-}\left(\mu \mathrm{g} \mathrm{N} \cdot \mathrm{L}^{-1}\right)$} \\
\hline & Mean & Min. & Max. & Std. Dev. & Mean & Min. & Max. & Std. Dev. \\
\hline REC CORREDOR & 30.51 & 0.00 & 350.00 & 64.21 & 28.39 & 0.00 & 83.64 & 21.81 \\
\hline ROGERA & 8.43 & 0.00 & 58.84 & 15.35 & 10.28 & 0.00 & 34.63 & 10.39 \\
\hline RIERETA & 14.52 & 0.00 & 117.26 & 26.08 & 8.76 & 0.00 & 30.57 & 10.11 \\
\hline LITORAL & 104.01 & 0.00 & 3155.92 & 539.65 & 4.84 & 0.00 & 18.53 & 4.73 \\
\hline CONECTADA & 17.10 & 0.00 & 474.60 & 82.31 & 8.56 & 0.23 & 74.33 & 14.26 \\
\hline E. TURIES & 1.66 & 0.00 & 15.96 & 3.99 & 5.83 & 0.00 & 33.55 & 7.47 \\
\hline \multirow[t]{3}{*}{ TAMARIU } & 8.49 & 0.00 & 240.35 & 41.77 & 5.50 & 1.04 & 27.11 & 6.42 \\
\hline & \multicolumn{4}{|c|}{$\mathrm{NO}_{3}^{-}\left(\boldsymbol{\mu g ~ N} \cdot \mathbf{L}^{-1}\right)$} & \multicolumn{4}{|c|}{$\operatorname{SRP}\left(\mu g P \cdot L^{-1}\right)$} \\
\hline & Mean & Min. & Max. & Std. Dev. & Mean & Min. & Max. & Std. Dev. \\
\hline REC CORREDOR & 8447.04 & 34.01 & 12388.6 & 3105.78 & 86.29 & 3.32 & 451.71 & 78.17 \\
\hline ROGERA & 262.01 & 0.00 & 1515.52 & 455.06 & 10.44 & 0.00 & 54.09 & 10.82 \\
\hline RIERETA & 144.11 & 0.00 & 281.02 & 276.27 & 32.77 & 1.32 & 139.83 & 34.72 \\
\hline LITORAL & 64.18 & 0.00 & 709.06 & 156.89 & 232.67 & 0.00 & 2278.82 & 433.16 \\
\hline CONECTADA & 60.54 & 0.00 & 566.60 & 144.14 & 14.04 & 0.00 & 58.20 & 17.79 \\
\hline E. TURIES & 26.62 & 0.00 & 265.92 & 67.86 & 25.99 & 0.00 & 89.84 & 23.02 \\
\hline TAMARIU & 6.77 & 0.00 & 187.43 & 32.60 & 17.82 & 0.00 & 47.49 & 13.61 \\
\hline
\end{tabular}

más próximos a la fuente de agua dulce. Durante esta situación dominada por el excedente de nitrato, las concentraciones de SRP son inapreciables exceptuando el pico observado inmediatamente después del temporal.

Cuando la tasa de renovación del agua disminuye, en nuestro caso, cuando el caudal del "Rec Corredor" no es suficiente como para enviar sus aguas a la marisma (finales de invierno), las lagunas o depresiones empiezan un período de confinamiento caracterizado por una rápida pérdida de nitrogeno inorgánico disuelto, y por un aumento de la concentración de SRP, especialmente en la laguna "Litoral" .

Desde el punto de vista geográfico, se observa un gradiente descendente en la concentración de nitrato (Tabla 1) y en la relación DIN/SRP (Tabla 2), en el eje Sur-Norte, debido a que la entrada de agua dulce se produce por el sur de la marisma.

Para analizar la viabilidad del agua residual depurada para la inundación de la marisma se ha considerado la posibilidad de inundar el "Estany del Cortalet". El "Estany del Cortalet" es una laguna artificial, de profundidad máxima cercana a $1 \mathrm{~m}$, construido con una finalidad de educación ambiental por la dirección del Parque Natural, el año 1989. Está situado en un antiguo prado halófilo de inundación ocasional y se mantiene inun-

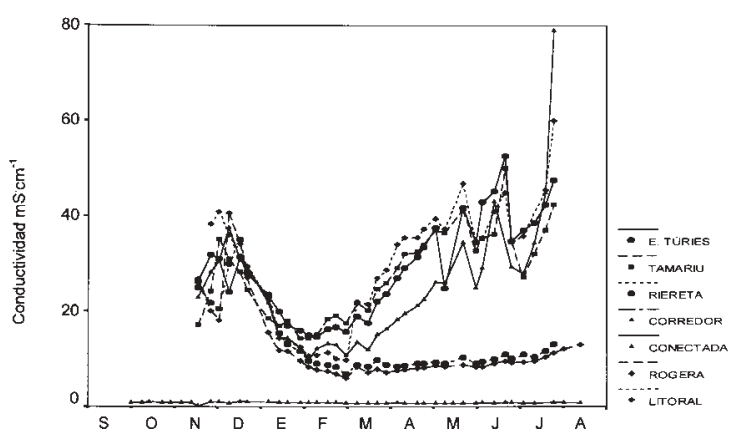

Figura 2. Variación temporal de la conductividad del agua $\left(\mathrm{mS} \mathrm{cm}^{-1}\right)$ en los puntos muestreados. Temporal variation of water conductivity $\left(\mathrm{mS} \mathrm{cm}^{-1}\right)$ in sampling sites. 

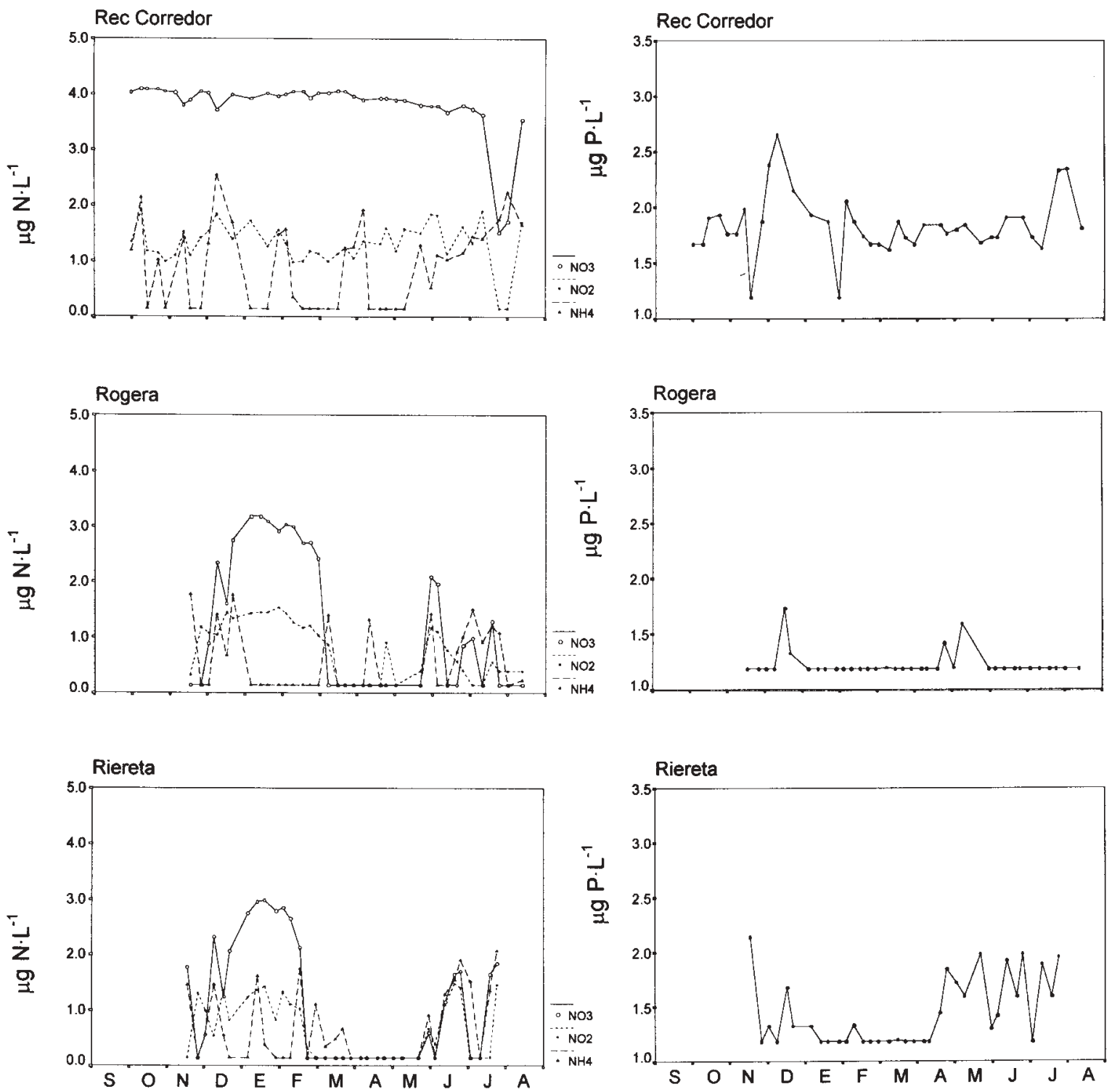

Figura 3. Variación temporal de las concentraciones (logaritmo decimal) en $\mathrm{mg} \mathrm{L}^{-1}$, de amonio, nitrito, nitrato (izquierda) y fósforo reactivo soluble (derecha), de los puntos de muestreo del sector sur de la marisma. Temporal variation of the concentrations of ammonium, nitrite, nitrate (left) and soluble reactive phosphorus (right) in $\mathrm{mg} \mathrm{L}^{-1}$ (decimal logarithm) of southern samples sites in the marsh.

dado durante prácticamente todo el año mediante el bombeo de agua procedente del "Rec Corredor". En esta laguna el agua se renueva con rapidez y raras veces queda confinada. El excedente de agua que circula por la laguna inunda los prados halófilos adyacentes. Los valores medios observados de nutrientes disueltos en esta laguna se muestran en la Tabla 2.

Si comparamos las cargas de nutrientes que se espera obtener en el efluente de los SHC con las 
Quintana et al.

Table 2. Concentración de nitrógeno inorgánico disuelto (DIN), fósforo reactivo soluble (SRP) y relación DIN/SRP en los puntos muestreados. Concentration of dissolved inorganic nitrogen (DIN), soluble reactive phosphorus (SRP) and DIN/SRP ratios in sampling sites.

\begin{tabular}{lrrr}
\hline PUNTO & $\begin{array}{c}\text { DIN } \\
\boldsymbol{\mu g ~ N} \cdot \mathbf{L}^{-1}\end{array}$ & $\begin{array}{c}\text { SRP } \\
\boldsymbol{\mu g} \mathbf{~ P} \cdot \mathbf{L}^{-1}\end{array}$ & $\begin{array}{c}\text { DIN/SRP } \\
\text { mol N/ mol P }\end{array}$ \\
\hline Efluente EDAR & 23230.00 & 3060.00 & 16.81 \\
Rec Corredor & 8506.14 & 86.29 & 218.28 \\
Efluente SHC & 5700.00 & 14.00 & 901.53 \\
Estany Cortalet & 301.00 & $<6.00$ & 111.08 \\
Rogera & 280.72 & 10.44 & 59.54 \\
Riereta & 167.39 & 32.77 & 11.31 \\
Litoral & 173.03 & 232.67 & 1.65 \\
Conectada & 86.20 & 14.04 & 13.59 \\
Estany d'en Túries & 34.11 & 25.99 & 2.91 \\
Tamariu & 20.76 & 17.82 & 2.58 \\
\hline
\end{tabular}

de las aguas de la zona (Tabla 2) se observan dos aspectos a remarcar. En primer lugar las cargas de nutrientes del efluente de los SHC son similares, incluso inferiores, a las del "Rec Corredor". Sin embargo difieren mucho de las concentraciones observadas en la marisma, donde se observan valores especialmente inferiores de nitrógeno inorgánico disuelto. En segundo lugar existen diferencias acusadas en la relación DIN/SRP en función de la tasa de renovación del agua. Las aguas del "Rec Corredor" y del "Estany del Cortalet", con una elevada tasa de renovación del agua, presentan una relación DIN/SRP alta, como suele ser característica en las aguas dulces. Por el contrario, las aguas de la marisma, pobres en nitrógeno y ricas en fósforo, presentan una relación DIN/SRP invertida, durante los períodos de confinamiento.

\section{DISCUSIÓN}

\section{Dinámica de nutrientes en la marisma}

Antes de la instalación de la compuerta en el canal de desagüe Massona-Rec Sirvent, la hidrología de la zona de marismas dels "Aiguamolls de
l'Empordà" consistía básicamente en un llenado brusco de toda la zona con agua de mar debido a los temporales y un vaciado paulatino por evaporación e infiltración (Quintana, 1995; Quintana et al., 1998a). Pocos dias después del temporal, quedaban inundadas sólo las depresiones de menor cota de la zona, donde el agua permanecía confinada hasta el siguiente episodio de inundación o hasta su desecación. En estas condiciones, el nitrógeno inorgánico disuelto se pierde rápidamente debido a la elevada tasa de desnitrificación cuando hay condiciones de anoxia en el sedimento. Por el contrario, el fósforo queda retenido en el sedimento y se acumula, liberándose de nuevo al agua en condiciones de anóxia del sedimento. Este proceso, que se ha denominado confinamiento diferencial (Quintana et al., 1998a), da como resultado un sistema con unos valores de la relación DIN/SRP muy bajos, inferiores a 1, durante la mayor parte de año. En estas condiciones de baja tasa de renovación del agua, la dinámica de los nutrientes esta básicamente controlada por la actividad biológica y por las diferencias en la velocidad de reciclado de los nutrientes (Comín \& Valiela, 1991). En estas condiciones el nitrógeno actuaría de factor limitante de la producción primaria.

Con la instalación de la compuerta, y la consiguiente entrada continua de agua dulce durante los meses de invierno, se produce un aumento considerable en la tasa de renovación del agua en la zona, durante este período. En estas condiciones, los mecanismos biogeoquímicos internos, son insuficientes para alterar las concentraciones de nitrógeno y fósforo (Comín \& Valiela, 1991). Puesto que el agua dulce del "Rec Corredor" tiene un relación DIN/SRP muy elevada (Tabla 2), los valores de esta relación encontrados en la marisma durante el invierno, son muy superiores a los esperados sin una entrada continua de agua dulce con las características citadas. Por otro lado esta aportación continuada supone un aumento neto de los nutrientes que entran en la marisma, provocando un aumento del grado de eutrofia (Quintana, 1995).

Si se comparan los valores de nutrientes obtenidos con los resultados de estudios anteriores realizados en la marisma (López \& Tomás, 1989; 

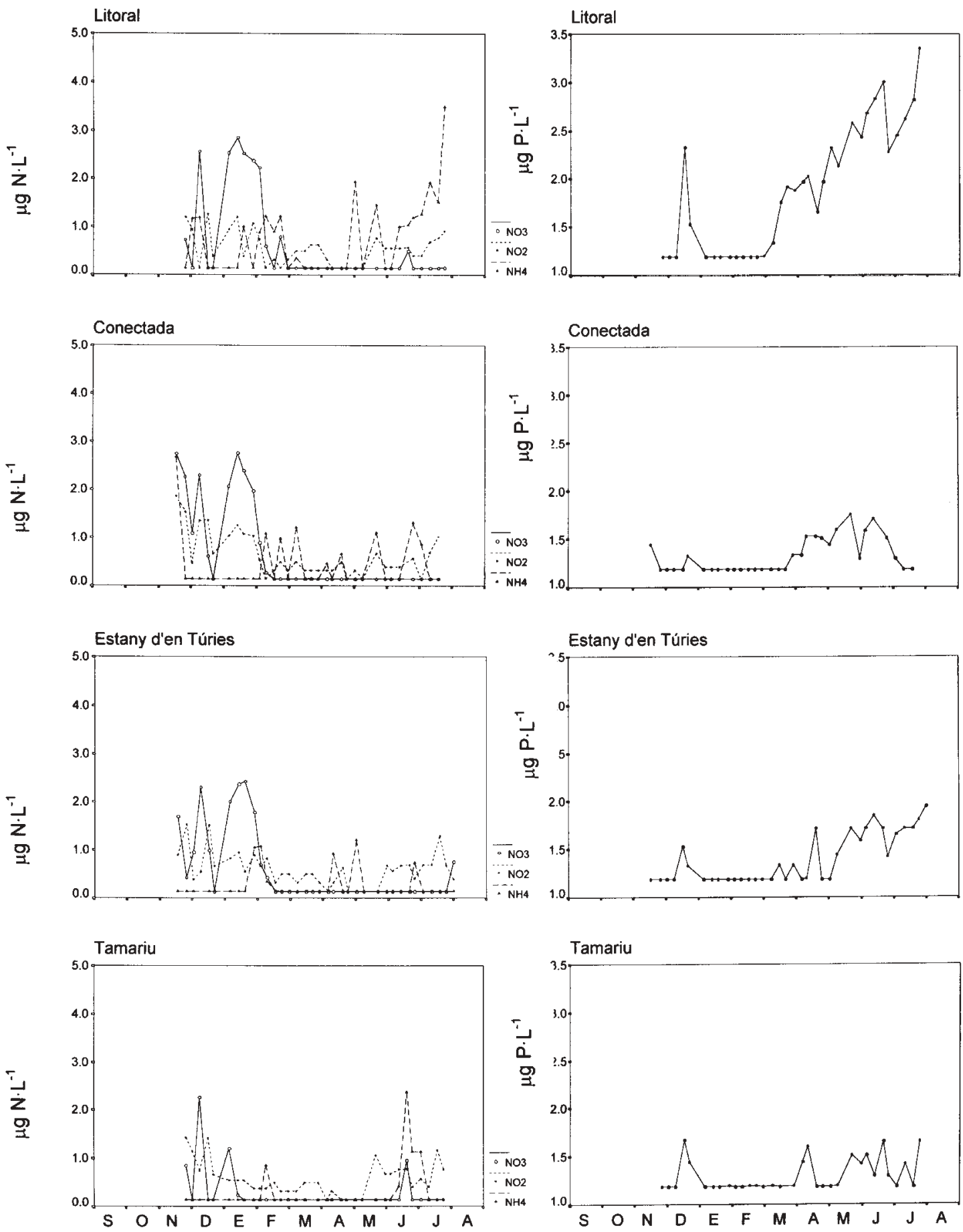

Figura 4. Variación temporal de las concentraciones (logaritmo decimal) en $\mathrm{mg} \mathrm{L}^{-1}$, de amonio, nitrito, nitrato (izquierda) y fósforo reactivo soluble (derecha), de los puntos de muestreo del sector norte de la marisma. Temporal variation of concentrations of ammonium, nitrite, nitrate (left) and soluble reactive phosphorus (right) in $\mathrm{mg} \mathrm{L}^{-1}$ (decimal logarithm) of northern sector sampling sites in the marsh. 
Quintana et al.

Table 3. Concentraciones medias de las formas inorgánicas solubles de nitrógeno, fósforo reactivo soluble $\left(\mu \mathrm{g} \cdot \mathrm{L}^{-1}\right)$ y $\mathrm{clorofila}\left(\mu \mathrm{g} \cdot \mathrm{L}^{-1}\right)$, en cinco puntos de muestreo de la marisma, a lo largo de distintos años. Datos de 1984 según López \& Tomás (1989), y datos de 1989-1990 según Quintana (1995). Mean concentration of the different forms of dissolved inorganic nitrogen, soluble reactive phosphorus ( $\left.\mu g \cdot L^{-1}\right)$ and chlorophyll $\left(\mu g \cdot L^{-1}\right)$, in five sampling sites across the marsh, in three different years. Data from 1984 according to López \& Tomás (1989) and data from 1989-1990 according to Quintana (1995).

\begin{tabular}{|c|c|c|c|}
\hline LITORAL & 1984 & $1989 / 90$ & 1996/97 \\
\hline $\mathrm{NH}_{4}$ & & $76.58(0-416.22)$ & $104.01(0-3155.92)$ \\
\hline $\mathrm{NO}_{2}^{4}$ & & $2.66(0-28.84)$ & $4.84(0-18.53)$ \\
\hline $\mathrm{NO}_{3}^{2}$ & & $13.44(0-170.38)$ & $64.18(0-709.66)$ \\
\hline SRP & & $323.95(0-1308.82)$ & $232.67(0-2278.82)$ \\
\hline Chla & & $21.62(0.25-75.52)$ & $24.85(1.67-98.48)$ \\
\hline CONECTADA & 1984 & 1989/90 & 1996/97 \\
\hline $\mathrm{NH}_{4}$ & & $49.56(0-447.44)$ & $17.10(0-474.6)$ \\
\hline $\mathrm{NO}_{2}^{4}$ & & $2.8(0-50.96)$ & $8.56(0.23-74.33)$ \\
\hline $\mathrm{NO}_{3}^{2}$ & & $16.66(0-137.2)$ & $60.54(0-566.60)$ \\
\hline SRP & & $50.84(0-177.94)$ & $19.04(0-58.20)$ \\
\hline Chla & & $8.24(0.25-51.99)$ & $11.87(3.75-60.23)$ \\
\hline TAMARIU & 1984 & 1989/90 & $1996 / 97$ \\
\hline $\mathrm{NH}_{4}$ & & $51.08(0-346.5)$ & $8.49(0-240.35)$ \\
\hline $\mathrm{NO}_{2}^{4}$ & & $1.96(0-10.64)$ & $5.50(1.04-27.11)$ \\
\hline $\mathrm{NO}_{3}^{2}$ & & $17.5(0-117.46)$ & $6.77(0-187.43)$ \\
\hline SRP & & $25.42(0-91.14)$ & $17.82(0-47.49)$ \\
\hline Chla & & $13.34(0.25-96.19)$ & $19.02(2.78-73.14)$ \\
\hline E. TÚRIES & 1984 & $1989 / 90$ & 1996/97 \\
\hline $\mathrm{NH}_{4}$ & - & & - \\
\hline $\mathrm{NO}_{2}$ & $7.21(0-28.14)$ & & $5.83(0-33.55)$ \\
\hline $\mathrm{NO}_{3}^{2}$ & $30.76(4.9-101.36)$ & & $26.62(0-265.92)$ \\
\hline SRP & $622.48(57.04-2055.9)$ & & $25.99(0-89.84)$ \\
\hline Chla & - & & - \\
\hline ROGERA & 1984 & $1989 / 90$ & $1996 / 97$ \\
\hline $\mathrm{NH}_{4}$ & - & & - \\
\hline $\mathrm{NO}_{2}^{4}$ & $2.08(0-7)$ & & $10.28(0-34.63)$ \\
\hline $\mathrm{NO}_{3}^{2}$ & $7.47(0-16.8)$ & & $262.01(0-1515.52)$ \\
\hline SRP & 89.49 (39.06-168.95) & & $10.44(0-54.09)$ \\
\hline Chla & - & & - \\
\hline
\end{tabular}

Quintana et al., 1998a) (Tabla 3), se observa una tendencia al incremento en la concentración de las formas inorgánicas de nitrógeno y a la disminución de la concentración de SRP, con la única excepción del "Estany d'en Túries", que mantiene valores medios similares. Se observa también un cierto incremento en la concentración de clorofila, aunque no se dispone de valores de todas las lagunas (Tabla 3). En la marisma, la entrada de nutrientes al sistema se produce por pulsos cuando hay entrada de agua y la concentración de nutrientes disueltos disminuye a lo largo del 
tiempo después de las aportaciones (Quintana et al., 1998a). Así, la variabilidad estocástica asociada al muestreo hace difícil la comparación entre estudios realizados en años diferentes y, si nos restringimos a los datos de la tabla 3 , únicamente es factible una interpretación especulativa de las diferencias observadas.

Una primera interpretación podría ser que, a partir del cambio en la hidrología causado por la regulación de flujos, se haya dado una sustitución del fósforo por el nitrógeno en el papel de nutriente limitante de la producción. Esta hipótesis parece poco verosímil si se tiene en cuenta que los nutrientes inorgánicos son, en condiciones estables, el excedente no incorporado por los organismos, y una sustitución del fósforo por el nitrógeno debería reflejarse en valores superiores a 16:1 en la relación DIN/SRP. Por otro lado, la retención de fósforo y las pérdidas de nitrógeno por desnitrificación hacen imposible esta sustitución cuando hay un alto grado de confinamiento y la limitación por fósforo podría darse, en el mejor de los casos, durante el invierno coincidiendo con la mayor renovación del agua (Fig. 5).

Una segunda hipótesis sería considerar que estas diferencias son el resultado de aportaciones importantes de agua rica en nitrógeno, que causan un aumento del grado de eutrófia. Esto implicaría un incremento neto de materia orgánica y de nutrientes totales (no se dispone de datos que puedan confirmar este punto) y un mayor consumo de SRP coincidiendo con las aportaciones, que explicaría la reducción en su concentración. El incremento en la concentración media de clorofila, los resultados obtenidos a partir de los organismos (Moreno-Amich et al., este volumen pág. 17) y la discusión, realizada anteriormente, de las variaciones estacionales de los nutrientes están también de acuerdo con esta interpretación. El incremento de clorofila no es muy notable, debido probablemente a la facilidad con que la transferencia de energía circula por la vía detrítica en estos ambientes cuando se produce un incremento de la materia orgánica disponible (Quintana, 1995).

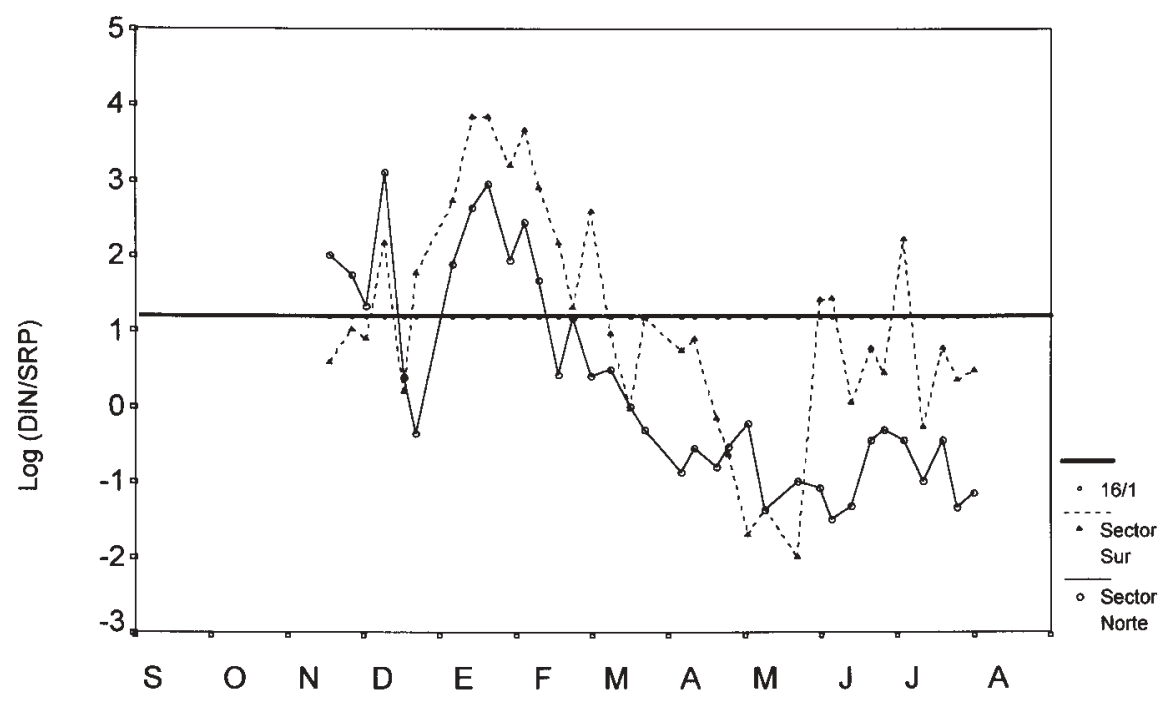

Figura 5. Variación temporal de la media de la relación DIN/SRP (logaritmo decimal) de los puntos correspondientes a los dos sectores de la marisma. Temporal variation of the logged DIN/SRP ratio both in southern and northern areas of the marsh (values are means of points in each sector). 


\section{Viabilidad de la utilización del efluente de los SHC}

La comparación de las concentraciones de nutrientes en las lagunas estudiadas con las concentraciones que se espera obtener en el efluente de los SHC (Tabla 2), muestran que la eliminación esperada de nitrógeno es claramente insuficiente para vertir el agua depurada a la marisma. Por otro lado las diferencias en la relación DIN/SRP indican que es prácticamente imposible obtener una agua de calidad adequada para la marisma, puesto que el efluente depurado será siempre deficitario en fósforo y las aguas de la marisma lo son en nitrógeno. Puesto que todas las depresiones de la marisma tienen el mismo comportamiento, ninguna de ellas podría asumir el efluente depurado.

Otro inconveniente que presentaría la inundación de la marisma con agua depurada, sería que las aportaciones se realizarían de manera continua y permanente, sobre un sistema adaptado a aportaciones puntuales. Las aportaciones máximas se producirían durante la época estival, hecho que invertiría el régimen hidrológico normal del sistema y, a la vez, evitaría la desecación estival característica y necesaria de un sistema de marismas mediterraneo.

Por el contrario la composición de nutrientes esperada del efluente de los SHC es muy similar a la composición del "Rec Corredor", única fuente de alimentación del "Estany del Cortalet" (Tabla 2). Los valores de la relación DIN/SRP en el "Estany del Cortalet" son también similares a los del agua depurada. Por consiguiente, se propone vertir el efluente de los SHC al "Estany del Cortalet", aprovechando los escorros naturales ya existentes. De esta manera, el "Estany del Cortalet", una laguna ya artificial, sería el único receptor de los efluentes de los SHC, manteniendo la zona de marisma en su estado natural y sin riesgo de eutrofización.

\section{AGRADECIMENTOS}

Este trabajo ha sido financiado parcialmente por el proyecto CICYT HID96-0916 "Fluctuaciones de alta frecuencia y tendencia del sistema de la marisma, en los "Aiguamolls de l'Empordà". Influencia de la gestión hídrica, efecto del control de mosquitos y viabilidad de la reinundación con aguas depuradas." y por el convenio con el Consorcio de la Costa Brava para la monitorización de la calidad de las aguas que circulan por el sistema de Aiguamolls de l'Empordà.

\section{BIBLIOGRAFIA}

CERDÀ, A., M. T. OMS, R. FORTEZA \& V. CERDÀ. 1996. Speciation of nitrogen in wastewater by flow injection. Analyst, 121: 13-17.

COMÍN, F. A. \& I. VALIELA. 1993. On the controls of phytoplankton abundance and production in coastal lagoons. Journal of Coastal Research, 9(4): 895-906.

MORENO-AMICH, R., X. QUINTANA, L. SUÑER, R. TROBAJO, S. GASCÓN, R. BARRERA, J. GIFRE \& M. MARTINOY. 1998. Monitorización de la calidad ecológica de las aguas y seguimiento de la composición de la comunidad d'invertebrados acuáticos en la zona del Parque Natural de Aiguamolls de l'Empordà. In: CD-ROM Compendio de informes correspondientes al "Seguimiento de la calidad de les aguas a la zona del Parque Natural de Aiguamolls de l'Empordà." Consorcio de la Costa Brava, Girona.

QUINTANA, X. 1995. Fluctuacions a la maresma dels Aiguamolls de l'Empordà $i$ estructura de la comunitat biològica. Tesis doctoral. Universitat de Girona.

QUINTANA, X., R. MORENO-AMICH. \& F. A. COMÍN. 1998a. Nutrient and plankton dynamics in a Mediterranean salt marsh dominated by incidents of flooding. Part 1: Differential confinement of nutrients. Journal of Plankton Research, 20(11): 2089-2107.

QUINTANA, X., F. A. COMÍN \& R. MORENOAMICH. 1998b. Nutrient and plankton dynamics in a Mediterranean salt marsh dominated by incidents of flooding. Part 2: Response of the zooplankton community to disturbances. Journal of Plankton Research, 20(11): 2109-2127.

RUZICKA, J. \& E. H. HANSEN. 1981. Flow Injection Analysis. John Wiley \& Sons. New York. 207 pp.

SARGATAL, J.1989. El Parc Natural: breu descripció geogràfica. In: Els Aiguamolls de l'Empordà. 
Aspectes ecològics, històrics $i$ socials. J. Sargatal \& J. Fèlix (eds.): 11-18. Quaderns dels Indiketes núm. 3. ART-3 Carles Vallès editor. Figueres.

SOLÓRZANO, L. 1969. Determination of ammonia in natural waters by the phenolhypochlorite method. Limnol. Oceanogr., 14: 799-801.
TALLING, J. F. \& D. DRIVER. 1963. Some problems in the estimation of chlorophyll $a$ in phytoplankton. Proc. Conf. on Primary Productiviy Measurements, Marine and Freshwater, Univ. Hawaii, 1961. U.S. Atomic Energy Comm, TID-7633: 142-146. 